\title{
Le rôle des croyances et des idéologies dans l'économie politique des réformes
}

Gilles Saint-Paul

Paris Sciences Economiques et Université de Toulouse I

\section{Introduction}

Depuis des décennies, nombre d'économistes se rêvent dans le rôle de conseiller du prince et prônent des mesures de bon sens qui amélioreraient le fonctionnement $d u$ marché du travail. Malgré leur implication croissante dans la vie publique, l'évolution de ce marché n'est guère favorable. Le chômage se stabilise à un niveau relativement élevé. Sa durée est importante et il frappe particulièrement les jeunes et les personnes âgées.

La flexibilité du marché du travail se heurte à une opposition politique importante. Les dernières mesures réellement favorables à la flexibilité remontent à l'abolition de l'autorisation administrative de licenciement en 1986 (mesure dont certains affirment que ses effets furent pervers dans la pratique, en renforçant le pouvoir discrétionnaire des tribunaux). Depuis, on assiste à des tentatives souvent infructueuses de flexibiliser le marché du travail à la marge. Ces tentatives se heurtent à une opposition violente (CIP, CPE...). L'idée d'un contrat unique plus flexible que le CDI actuel est louable mais aucun homme politique ne s'aventure à la proposer.

Si la réforme du marché du travail est plus difficile en France qu'ailleurs, c'est en grande partie à cause de perceptions populaires négatives sur l'économie de marché. Il existe un système de croyances qui augmente les résistances aux réformes. Ces croyances se reproduisent par le biais des institutions - le système éducatif et la presse. En 2006, un sondage international a demandé à des ressortissants de vingt pays développés et en voie de développement leurs opinions sur l'économie de marché. 
Les résultats sont reproduits ci-dessous :

\section{Tableau 1 : Les attitudes sur l'économie de marché en France et ailleurs.}

at. The free enterprise system and free market economy is the best system on which to base the future of the world.

\begin{tabular}{l|cccccccc} 
& $\begin{array}{c}\text { Total } \\
\text { Agree }\end{array}$ & $\begin{array}{c}\text { Total } \\
\text { Disagree }\end{array}$ & $\begin{array}{c}\text { Strongly } \\
\text { Agree }\end{array}$ & $\begin{array}{c}\text { Somewhat } \\
\text { Agree }\end{array}$ & $\begin{array}{c}\text { Somewhat } \\
\text { Disagree }\end{array}$ & $\begin{array}{c}\text { Strongly } \\
\text { disagree }\end{array}$ & $\begin{array}{c}\text { Depends } \\
\text { / Neither }\end{array}$ & $\begin{array}{c}\text { DK/ } \\
\text { Refused }\end{array}$ \\
\hline Argentina & 42 & 29 & 11 & 31 & 13 & 16 & 2 & 26 \\
Brazil & 57 & 30 & 18 & 40 & 19 & 11 & 4 & 9 \\
Canada & 65 & 29 & 22 & 43 & 19 & 10 & 2 & 3 \\
China & 74 & 20 & 25 & 49 & 16 & 4 & 3 & 4 \\
France & 36 & 50 & 10 & 26 & 27 & 23 & 3 & 11 \\
Germany & 65 & 32 & 29 & 36 & 24 & 9 & 2 & 1 \\
Great Britain & 66 & 27 & 26 & 39 & 17 & 10 & 2 & 5 \\
India & 70 & 17 & 34 & 35 & 12 & 6 & 3 & 11 \\
Indonesia & 68 & 29 & 22 & 46 & 25 & 4 & 1 & 3 \\
Italy & 59 & 31 & 21 & 38 & 20 & 12 & 4 & 5 \\
Kenya & 59 & 25 & 33 & 25 & 12 & 13 & 3 & 13 \\
Mexico & 61 & 38 & 21 & 40 & 27 & 11 & 0 & 1 \\
Nigeria & 66 & 29 & 34 & 31 & 13 & 16 & 2 & 4 \\
Philippines & 73 & 22 & 23 & 50 & 17 & 5 & 2 & 4 \\
Poland & 63 & 19 & 22 & 41 & 14 & 5 & 4 & 14 \\
Russia & 43 & 34 & 11 & 32 & 25 & 9 & 7 & 15 \\
S. Korea & 70 & 19 & 11 & 60 & 17 & 2 & 5 & 6 \\
Spain & 63 & 28 & 27 & 36 & 14 & 14 & 1 & 8 \\
Turkey & 47 & 36 & 5 & 42 & 31 & 6 & 4 & 12 \\
USA & 71 & 24 & 34 & 37 & 15 & 9 & 2 & 3 \\
\hline Average & $\mathbf{6 1}$ & $\mathbf{2 8}$ & $\mathbf{2 2}$ & $\mathbf{3 9}$ & $\mathbf{1 9}$ & $\mathbf{1 0}$ & $\mathbf{3}$ & $\mathbf{8}$
\end{tabular}

Source : http://65.109.167.118/pipa/pdf/jan06/FreeMarkets_Jan06_quaire.pdf 
On constate que la France a été le seul pays où une majorité de sondés se déclaraient hostile à celle-ci. L'échantillon comprenait pourtant des pays comme l'Argentine ou le Brésil qui ont pâti de mouvements brutaux de capitaux, ou la Russie qui a vécu une transition difficile vers l'économie de marché.

Dans cet article, nous essayons de comprendre la persistance de croyances potentiellement biaisées, notamment à l'encontre de l'économie de marché. Nous analysons d'abord les incitations, pour certains groupes d'intérêts (notamment les «insiders » du marché du travail représentés par les principaux syndicats), de favoriser certaines croyances et idéologies parce que celles-ci conduisent à des décisions qui leur sont favorable. Nous montrons ensuite comment, même en l'absence de manipulation, des croyances erronées peuvent se propager à travers le choix de carrière des individus.

\section{La manipulation idéologique des croyances dans un monde incertain}

Une première source d'idéologies «hostiles » à l'économie de marché est bien entendu la propagande. Cet aspect des choses soulève bien des problèmes. Il conviendrait de s'interroger sur ce qui fait qu'une information, une rumeur, ou une vision du monde est « crue » et tend à se propager dans la population.

Les économistes ont développé des théories qui interprètent ces phénomènes en termes de cascades informationnelles ${ }^{1}$. L'idée est que si un agent observe l'adhésion d'un autre agent à une croyance (à travers ses actions ou l'expression de ses opinions), il en déduit que cet agent possède une information privée qui augmente la probabilité que cette opinion soit valide, d'où une incitation à adopter les mêmes croyances et les mêmes comportements. Dans ces modèles, aucun participant n'a intérêt à manipuler l'information. La cascade informationnelle peut créer des inefficacités, au sens où un agent qui apprend par lui-même plutôt que de s'en tenir à la rumeur ou au consensus, exerce une externalité positive sur ceux qui l'imiteront. A

\footnotetext{
${ }^{1}$ Voir par exemple Banerjee, Abhijit V, 1992. "A Simple Model of Herd Behavior," Quarterly Journal of Economics, MIT Press, vol. 107(3), pages 797-817
} 
cause de cette externalité, on peut observer des phénomènes de type «moutons de panurge » où tous les agents adoptent une croyance erronée parce qu'une chaîne de transmission de cette croyance s'est établie à partir d'un individu qui se trompait. Il ne reste pas moins qu'en moyenne, il n'y a aucun biais systématique et que de telles chaînes sont d'autant moins probables que la croyance considérée est éloignée de la réalité. Les choses sont différentes quand les croyances ont un effet systémique, par exemple sur les prix de marché, et qu'il est possible de les manipuler par des annonces. Bien qu'une annonce fausse a un impact négatif sur la réputation de l'annonceur, ce qui réduit considérablement la possibilité de manipulation à long terme, Bénabou et Laroque $(1992)^{2}$ ont montré que la manipulation de l'information peut être persistante si celle-ci est considérée comme imparfaite par le public qui ne peut faire la différence entre une erreur d'une annonceur honnête et une authentique manipulation. Ce type de modèle, qui s'applique aux marchés financiers, n'explique cependant pas pourquoi un biais systématique peut exister dans les croyances des agents. La manipulation résulte de l'information privée du manipulateur, et celle-ci varie au cours du temps et diffère de l'information publique de façon non systématique.

Il est intéressant de montrer, dans le contexte de l'économie politique des réformes du marché du travail, qu'un groupe d'intérêt peut vouloir manipuler les croyances dans une direction systématique, alors même qu'il ne dispose d'aucune information privée.

Prenons un exemple simple fondé sur le salaire minimum³ ${ }^{3}$ Supposons qu'il existe trois groupes de travailleurs :

- les plus qualifiés, dont le travail est complément avec celui des moins qualifiés dans la fonction de production ;

- les moins qualifiés ;

\footnotetext{
2 "Using Privileged Information To Manipulate Markets: Insiders, Gurus And Credibility", Quarterly Journal of Economics, 107 (1992), 921-948

${ }^{3}$ Pour une discussion plus complète, voir G. Saint-Paul, "Why are European countries diverging in their unemployment experience", Journal of Economic Perspectives, 18, 4, 2004, 49-68
} 
- les travailleurs «intermédiaires », qui sont parfaitement substituables aux moins qualifiés mais dont la productivité est plus élevée. Pour fixer les idées, on peut supposer que la productivité d'un travailleur intermédiaire est de $50 \%$ plus élevée que celle d'un moins qualifié.

A l'équilibre du marché du travail, ces travailleurs seront payés leur productivité marginale. En particulier, le salaire des travailleurs intermédiaires sera égal à celui des moins qualifiés, plus cinquante pour cent. Si les qualifiés ne sont pas trop nombreux, leur salaire sera également plus élevé que celui des moins qualifiés, voire des travailleurs intermédiaires.

Supposons maintenant que cette société vote sur l'instauration d'un salaire minimum supérieur de $10 \%$ au salaire d'équilibre des moins qualifiés. Quel est l'effet de cette réforme sur le bien-être des différentes catégories de travailleurs ? Les moins qualifiés coûtent désormais plus que leur salaire. Les entreprises réagissent en les licenciant. Cela réduit la quantité du facteur «travailleurs moins qualifiés + travailleurs intermédiaires » utilisé dans le processus de production. La productivité marginale de ce facteur augmente, tandis que celle du facteur complémentaire (les plus qualifiés) diminue. Le processus d'ajustement s'arrête lorsque la productivité marginale des moins qualifiés est remontée au niveau du salaire minimum. Dans la nouvelle situation :

- Le salaire des moins qualifiés a augmenté de $10 \%$, mais certains d'entre eux ont perdu leur emploi ;

- Le salaire des plus qualifiés a baissé, car leur productivité est diminuée de par la plus grande rareté de l'input complémentaire.

- Le salaire des travailleurs intermédiaires a également augmenté de $10 \%$, car l'arbitrage entre employer un intermédiaire ou un moins qualifié implique que le salaire des premiers doit rester égal à celui des seconds, plus cinquante pour cent. En outre, aucun d'entre eux n'a perdu son emploi. 
Comment chacun de ces groupes va-t-il voter? Pour le savoir, les plus qualifiés et les travailleurs intermédiaires n'ont besoin que du raisonnement qualitatif que nous venons de décrire. Les plus qualifiés savent que la mesure leur est nuisible, les travailleurs intermédiaires savent qu'ils y gagnent. En revanche, les moins qualifiés doivent évaluer l'effet de la mesure sur la probabilité qu'ils conservent leur emploi, qui dépend de l'élasticité de la demande de travail par rapport au salaire. Si cette élasticité est faible (inférieure à 1 si les moins qualifiés maximisent simplement leurs revenus espérés), le risque de perte d'emploi est faible au regard de la hausse de salaire anticipée, et les travailleurs moins qualifiés voteront pour le salaire minimum. Si elle est forte, ils voteront pour le salaire minimum.

Il en résulte qu'un organe médiatique qui représente les travailleurs intermédiaires, aura toujours intérêt à propager l'idée que cette élasticité est faible, dans le but d'inciter les travailleurs moins qualifiés à voter en faveur du salaire minimum. Comme ces travailleurs bénéficient de la mesure indépendamment de la valeur réelle de cette élasticité, cette incitation existe quelle que soit cette valeur et quelle que soit l'information dont ils disposent. Inversement, les travailleurs qualifiés ont intérêt à propager l'idée que cette élasticité est élevée.

Bien que l'idée d'une relation négative entre coût du travail et demande de travail ne fasse l'objet d'aucune controverse dans la plupart des pays, ce n'est pas le cas en France, comme en témoigne la fameuse «affaire Laroque-Salanié » au cours de laquelle des syndicats avaient protesté contre la publication d'une étude scientifique parce que celle-ci impliquait que le salaire minium détruisait plusieurs centaines de milliers d'emplois (voir encadré 1). Cet épisode permet d'être mieux compris dans le cadre d'analyse que nous venons de décrire.

Encadré $1:$ la lettre ouverte des syndicats de l'Insee à la direction après la publication de l'article de Laroque et Salanié 
" En plein débat social sur le chômage, un nouvel article paru dans le numéro 331 d'Economie et Statistique, écrit par MM. Laroque et Salanié, a donné lieu à une désastreuse reprise de presse : "Près d'un chômeur sur deux ne veut pas travailler, selon une étude de l'INSEE " La Tribune, "SMIC et 35 Heures : l'INSEE jette un pavé dans la mare " Le Figaro Economie, "L'Insee a calculé qu'une hausse de 10\% du SMIC détruirait 290000 emplois, rappelle le Medef "Le Monde Economie, etc...

Ainsi l'INSEE en tant que tel se trouve mis en avant pour des jugements péremptoires sur les chômeurs, les 35 heures, l'existence et le niveau du SMIC.

L'opportunité de publier cet article avait été débattue et tranchée au sein de la direction de I'INSEE. En décidant cette publication, celle-ci ne pouvait ignorer l'impact médiatique qu'elle provoquerait.

Le fait que l'article soit signé par Guy Laroque en qualité de directeur à l'INSEE et non de chercheur, ainsi que le contenu du "chapeau" figurant en tête de l'article, ne peuvent laisser aucun doute sur le but recherché.

De ce fait, c'est bien l'INSEE qui se trouve engagé au travers de cet article d'Economie et Statistique, quelques soient les précautions formelles indiquant que les points de vue exprimés n'engagent que leurs auteurs.

Or cet article, qui aurait été refusé par plusieurs revues internationales, est profondément contestable:

- il utilise un modèle qui laisse supposer que la protection sociale est un encouragement à la fainéantise et qu'une partie importante des sans emplois ne "valent " pas le SMIC (en particulier " les femmes en couple qui ont un diplôme inférieur au baccalauréat ") ;

- il avoue que "le SMIC ne peut avoir, dans ce modèle, que des effets négatifs sur l'emploi ";

- il néglige superbement la confusion inévitable qu'il introduit chez le lecteur entre " non-emploi " et " chômage ".

Au total, ce texte présente une vision totalement unilatérale de la société et ses auteurs se sont dispensés de tout retour au réel.

Avec la publication de telles affirmations et prises de position, la direction de l'institut porte un coup important à l'indépendance et à la crédibilité scientifique de l'INSEE et. à son indépendance.

Elle déroge aux engagements pris dans le passé par la direction, après une affaire comparable. Rappelons le voeu du Comité Technique Paritaire de l'INSEE du 13/11/90 :

"En matière d'études ou de prévisions, l'INSEE doit s'efforcer de présenter plusieurs solutions ou scénarios permettant notamment d'apprécier la robustesse de ses travaux "

Le Comité de direction doit assumer ses responsabilités vis à vis de l'opinion publique et donc faire savoir que la publication de cet article a été une erreur.

Paris, le 20 juillet 2000 »

Source : $\quad$ http://cgtinsee.free.fr/dossiers/Travaux\%20Insee/etudes/2001\%20-

\%202005/Larosala.htm

La question de la « crédibilité » du manipulateur reste cependant entière : dans quelle mesure peut-on empêcher les agents d'apprendre graduellement la valeur 
réelle des paramètres sous-jacents qui gouvernent l'évolution de l'économie, ainsi que d'ignorer les idéologies qui contredisent leur expérience personnelle?

Une réponse évidente est que l'économie n'est pas une science exacte. Les études empiriques se contredisent, les modèles alternatifs ne sont pas nécessairement compatibles, et les paramètres que l'on cherche à évaluer ne sont que des représentations (contrairement, par exemple, au diamètre de la terre ou à la constante gravitationnelle). Plus une science est «molle» plus son objet est sujet à la manipulation à des fins politiques et il serait intéressant d'étudier les diverses formes que cette manipulation peut prendre. On peut propager des informations sur la valeur d'une corrélation ou d'une élasticité, mais il conviendrait de s'interroger également sur le rôle préemptif de la formulation même d'un problème sur la formation des croyances. Pour prendre un exemple simple, pendant des décennies l'enseignement de la macroéconomie s'est bien souvent borné à des modèles keynésiens de court terme où, par construction, la notion d'une contrainte budgétaire intertemporelle est exclue. Un tel cadre justifiait ensuite l'accumulation de déficits publics ainsi qu'un multiplicateur élevé de ces déficits, en l'absence d'équivalence ricardienne.

Une seconde réponse est que des capacités cognitives non négligeables sont nécessaires pour évaluer correctement ces paramètres. Dans l'exemple qui précède, le groupe social qui a besoin de la plus grande expertise pour formuler son vote est précisément celui dont on s'attend que les capacités cognitives soient les plus faibles : les travailleurs les moins qualifiés. Les croyances ne sont pas nécessairement formées de façon rationnelle sur la base d'études scientifiques. Les psychologues ont observé, par exemple, que l'on croyait d'autant plus à la validité d'une idée que celle-ci était répétée souvent.

Enfin, le processus d'apprentissage est freiné par le renouvellement des générations. Avant d'apprendre par l'expérience, les individus sont confrontés pendant leur enfance et leur adolescence à des notions qu'on leur inculque. On peut considérer ces notions comme leur tenant lieu de "priors» au sens Bayésien du terme. Ces croyances initiales sont ensuite révisées sous l'effet de l'expérience et des informations supplémentaires. Dans la section suivante, nous nous demandons dans 
quelle mesure le système éducatif peut transmettre des croyances biaisées, par exemple défavorables à l'économie de marché.

3. Endoctrinement endogène et autosélection des clercs

Il est naturel de supposer que l'hostilité à l'économie de marché est renforcée par les biais des agents qui jouent un rôle-clé dans la formation des croyances : les enseignants et les journalistes. Il n'est pas difficile de documenter ces biais. Le graphique suivant a été publié par Le Monde de l'Education à l'occasion des élections de 2002.

Tableau 2 : Une enquête sur le vote des enseignants en 2002

\section{INTENTION DE VOTE AU PREMIER TOUR DE L'ÉLECTION PRÉSIDENTIELLE}

\begin{tabular}{|l|l|}
\hline - Arlette LAGUILLER & 8 \\
\hline - Olivier BESANCENOT & 2 \\
\hline - Robert HUE & 3 \\
\hline - Jean-Pierre CHEVÈNEMENT & 16 \\
\hline - Lionel jospin & 29 \\
\hline - Noël MAMère & 14 \\
\hline - François bayrou & 3 \\
\hline - Jacques chirac & 23 \\
\hline
\end{tabular}




\begin{tabular}{|l|l|}
\hline - Alain madelin & 2 \\
\hline - Charles pasqua & 0 \\
\hline - Jean-Marie le pen & 0 \\
\hline - Bruno mégret & 0 \\
\hline
\end{tabular}

(N'ont pas exprimé d'intention de vote : $17 \%$ ).

Source : http://www.tns-sofres.com/etudes/pol/280302 enseignants r.htm

On note que la gauche totalise $59 \%$, l'extrême-gauche marxiste $13 \%$, la droite $28 \%$ et l'extrême-droite $0 \%$. Les opinions politiques des enseignants sont donc peu représentatives de celles de la population. Une enquête de l'hebdomadaire Marianne sur le vote des journalistes donnait des résultats similaires.

Ces résultats peuvent s'interpréter de façons diverses. On peut tout d'abord supposer que les «clercs » sont mieux informés sur la réalité objective, et que s'ils sont de gauche, c'est parce que c'est la «gauche qui a raison». On peut également penser à une corrélation positive entre le fait d'être de « gauche » donc « altruiste » et de vouloir " prêcher » et « répandre la bonne parole ».

Je voudrais attirer l'attention ici sur une autre hypothèse, qui explique les opinions des enseignants (mais pas celles des journalistes). Elle repose sur l'idée est qu'il est économiquement rationnel pour un individu qui a une opinion négative sur l'économie de marché de chercher un emploi dans un secteur protégé des forces du marché - donc en particulier dans la fonction publique, où officient les enseignants. En d'autres termes, la formation des croyances des nouvelles générations est confiée à une population dont les propres croyances reflètent un biais d'autosélection relativement à la distribution des croyances de l'ensemble de la population. Certes, si le contenu des programmes scolaires est décidé démocratiquement par les citoyens, l'école transmettra les croyances de l'électeur médian plutôt que celles de 
l'enseignant moyen. Mais ces dernières prennent un poids disproportionné dès lors que

(i) les programmes scolaires sont fixés par la bureaucratie du système éducatif, parce que celle-ci peut se protéger des pouvoirs publics.

(ii) plus simplement, il existe des difficultés de contrôle des enseignants qui conservent une marge de manœuvre pour transmettre leurs propres croyances.

On peut analyser les conséquences de ce phénomène dans le cadre d'un modèle théorique (décrit dans Saint-Paul, 2007) ${ }^{4}$. L'école et le milieu familial déterminent les priors (au sens Bayésien) des nouvelles générations. Ces croyances concernent les caractéristiques du marché du travail, telles que le profil des salaires en fonction de l'âge, de l'occupation, etc, ainsi que les transitions entre emploi, chômage, inactivité, et les caractéristiques non salariales de chaque emploi (pénibilité, etc). Ce sont ces variables qui sont pertinentes pour le choix de carrière de l'individu. D'une manière générale, un individu qui a une opinion peu favorable sur l'économie de marché aura des croyances plutôt négatives (au sens de l'utilité) sur ces variables. Dans le modèle, on n'en utilise qu'une seule : la "précarité », qui représente la fraction du temps passée au chômage par un individu qui travaille dans le secteur privé. On suppose qu'elle peut être élevée ou faible, et la croyance d'un individu est définie comme la probabilité $p$ qu'elle soit élevée. Plus $p$ est grand, plus l'individu est « hostile » à l'économie de marché.

Une fois leurs «priors » déterminés, les individus optent pour une carrière. Ils choisissent entre le secteur privé et le secteur public. S'ils travaillent dans le secteur public, ils deviennent enseignants, et jouissent de la sécurité de l'emploi, c'est-à-dire que la précarité est nulle. Cette hypothèse n'est pas indispensable, l'hypothèse importante est que la précarité dans le secteur public soit connue.

\footnotetext{
${ }^{4}$ « A theory of endogenous indoctrination », mimeo, à paraître.
} 
Un individu a d'autant plus d'incitations à être fonctionnaire qu'il pense que la précarité est élevée dans le secteur privé, en d'autres termes qu'il a une mauvaise opinion de l'économie de marché. La distribution des «priors» d'une nouvelle génération détermine donc la courbe d'offre de travail des fonctionnaires. A salaire des fonctionnaires donné, préfèrent être fonctionnaires les gens qui pensent que la précarité est plus élevée qu'un certain seuil. Cette relation est représentée sur la figure 1. Sur l'axe horizontal figure la variable $p$, qui est la probabilité perçue que la précarité soit élevée. Sur l'axe vertical figure le salaire des fonctionnaires $\omega$. Pour un salaire des fonctionnaires donné, tous les individus dont la perception est plus élevée que la valeur de $p$ correspondant à $\omega$ sur la courbe préfèrent être fonctionnaires, les autres préférant travailler dans le secteur privé.

Figure 1: l'offre de travail des fonctionnaires

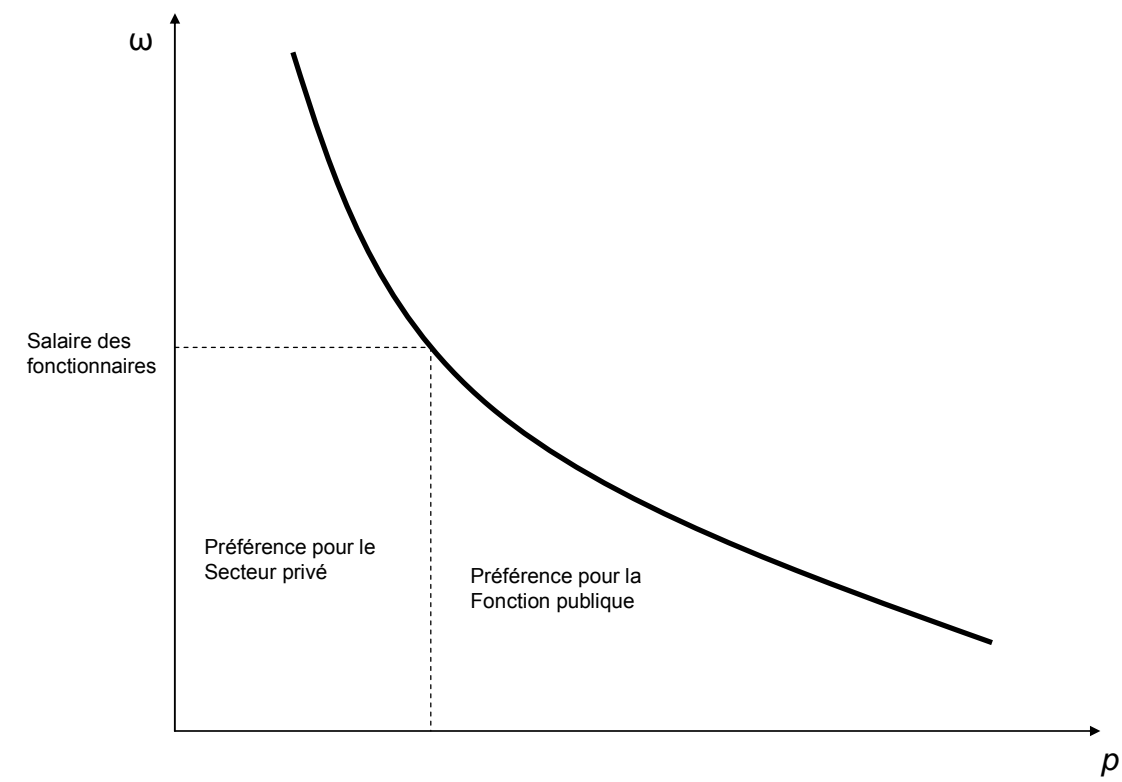


Il existe une demande inélastique de fonctionnaires : une fraction $n$ fixe de chaque cohorte devient fonctionnaire. Leur salaire s'ajuste de façon à égaliser l'offre et la demande. On peut noter, de manière intéressante, que plus les croyances sur l'économie de marché sont négatives, plus l'offre de fonctionnaires est élevée et plus leur salaire est faible ${ }^{5}$. Un gouvernement de type Léviathan aurait donc un intérêt stratégique - par exemple lorsqu'il fixe le contenu des manuels scolaires - à propager ce type de croyance, ce qui lui permettrait d'augmenter le nombre de fonctionnaires à budget donné ou de dégager des ressources qu'il pourrait redistribuer à sa clientèle. Cependant, ces considérations sont exclues ici, on considère que les enseignants transmettent leurs propres croyances, pas celle du gouvernement, et qu'enseignants et parents sont de bonne foi.

Empiriquement, le lien entre les opinions négatives sur l'économie de marché chez les enseignants et au sein du public, documentées ci-dessus, et l'offre de fonctionnaires est très plausible pour la France. Une enquête de l'Ifop montre en effet qu'à une large majorité, les jeunes qui sortent du système éducatif souhaitent être fonctionnaires :

\footnotetext{
${ }^{5}$ Le gouvernement peut cependant vouloir verser des rentes aux fonctionnaires, par exemple pour se constituer une clientèle politique. Une hausse de l'offre peut alors se traduire par un plus grand rationnement dans l'accès à la fonction publique plutôt que par une baisse du salaire.
} 
Tableau 3 : les souhaits de carrière des jeunes Français.

\section{Le souhait de travailler dans la fonction publique}

Question: Et vous personnellement, si vous en aviez la possibilité, souhaiteriez-vous travailler dans ... ?

TOTA Oui, Oui, NoTA probablem Non, dans la L oui certainem probablem $\mathrm{L}$ non en ent

ent

pas pas publiq

\begin{tabular}{|c|c|c|c|c|c|c|c|}
\hline$(\%)$ & $(\%)$ & $(\%)$ & $(\%)$ & $(\%)$ & $(\%)$ & $(\%)$ & ${ }^{(\%}(\%)$ \\
\hline $\begin{array}{l}\text { La } \\
\text { fonction } \\
\text { publique } \mathbf{7 0} \\
\text { en } \\
\text { général }\end{array}$ & 33 & 37 & 28 & 19 & 9 & 2 & -100 \\
\hline $\begin{array}{l}\text { La } \\
\text { fonction } \\
\text { publique } 55 \\
\text { territorial } \\
\text { e }\end{array}$ & 21 & 34 & 44 & 28 & 16 & 1 & -100 \\
\hline $\begin{array}{l}\text { La } \\
\text { fonction } \\
\text { publique } \\
\text { d'Etat }\end{array}$ & 25 & 27 & 47 & 26 & 21 & 1 & -100 \\
\hline $\begin{array}{l}\text { La } \\
\text { fonction } \\
\text { publique } \mathbf{4 8} \\
\text { hospitaliè } \\
\text { re }\end{array}$ & 22 & 26 & 51 & 25 & 26 & 1 & -100 \\
\hline
\end{tabular}

Source : http://www.ifop.com/europe/sondages/opinionf/jeunesfonctionpubl.asp 
Les travailleurs du secteur privé développent une trajectoire de carrière, perdant quelquefois leur emploi. Cette expérience leur permet de réviser leurs croyances et de les transmettre à leurs enfants. Les fonctionnaires n'ont pas cette expérience et leurs croyances révisées sont identiques à leurs croyances initiales. Ils la transmettent à tous les élèves dont ils ont la charge. Le «prior » d'un individu de la nouvelle génération est une combinaison linéaire de celui de son professeur et de celui de ses parents.

L'évolution des croyances de cette société subit l'effet de deux forces :

-D'une part, l'apprentissage bayésien de la précarité par les employés du secteur privé, apprentissage qui est transmis à leurs enfants.

-D'autre part, la transmission des croyances par les professeurs, qui reflète leur propre biais en faveur d'une économie précaire.

Par exemple, un employé qui n'a pas eu de chance dans sa carrière révisera ses croyances vers une précarité élevée. Son enfant qui hérite d'une partie de ces croyances aura une valeur de $p$ élevée, ce qui augmente la probabilité qu'il devienne professeur. Si c'est le cas, il contribuera à son tour à augmenter la valeur de $p$ dans la génération suivante, y compris parmi ceux qui deviendront professeurs. L'expérience malheureuse de cet employé affecte donc les croyances de beaucoup d'autres individus dans toutes les générations suivantes, à travers son effet sur le choix de carrière de son enfant.

En l'absence d'école, la transmission des croyances par la seule famille entraînerait une convergence graduelle de celles-ci vers la vraie valeur de la précarité. Si la précarité est réellement élevée, alors le biais de l'école accélère la vitesse de convergence de cette vraie valeur - les individus sont mieux informés, et prennent de meilleures décisions individuelles et collectives, qu'en l'absence d'école.

Si par contre la précarité est faible, le biais de l'école réduit la vitesse de convergence; dans certains cas, les croyances peuvent ne pas converger vers la vraie 
valeur de la précarité. Le système éducatif contribue alors au maintien permanent de croyances erronées dans la société, malgré la bonne foi des enseignants.

Nous donnons maintenant quelques illustrations numériques de ces résultats, en insistant sur deux paramètres importants :

- L'héritabilité : C'est le poids relatif de la famille dans la formation des croyances de l'enfant. Plus cette héritabilité est faible, plus le biais du système éducatif a d'impact sur les croyances des nouvelles générations.

- L'entropie sociale : C'est l'inverse de la prévisibilité du choix de carrière d'un individu en fonction de ses croyances initiales. Cette entropie est minimale - et nulle - si tous les individus situés au-dessus du seuil critique de $p$ au-delà duquel on préfère être fonctionnaire le deviennent effectivement, et si tous les individus situés en deçà de ce seuil travaillent dans le secteur privé. Elle est maximale si l'allocation des individus à leur carrière est indépendante de leurs croyances. On voit que plus l'entropie sociale est faible, plus les enseignants vont être «sélectionnés » relativement au reste de la population. Si l'entropie est maximale l'école ne présente aucun biais et la dynamique d'apprentissage est la même qu'en l'absence de système éducatif. Ce concept est proche, mais différent, de celui de mobilité sociale qui, ici, serait représenté par la probabilité d'être enseignant conditionnellement à ce que son parent ne le soit pas. Dans le modèle, les agents doivent investir des ressources pour tenter de devenir fonctionnaires - par exemple pour réussir un concours. La probabilité de réussite dépend de leur effort et ceux qui ont une valeur de $p$ plus élevée sont incités à fournir un effort plus grand. Plus la probabilité de réussite est élastique en fonction de l'effort, plus les fonctionnaires seront concentrés parmi les populations avec un $p$ élevés. L'entropie sociale est donc une fonction décroissante de l'élasticité de la probabilité de réussite par rapport à l'effort, appelée $\lambda$. Inversement, si cette élasticité est nulle, tout le monde 
fournira le même effort et la probabilité d'accéder à la fonction publique sera indépendante de $p-l$ 'entropie est alors maximale.

Dans les simulations qui suivent, nous supposons que l'économie de marché est objectivement une «bonne chose », au sens où c'est la valeur faible de la précarité qui prévaut dans la réalité. Cela signifie que les croyances sont correctes lorsque $p=$ 0 .

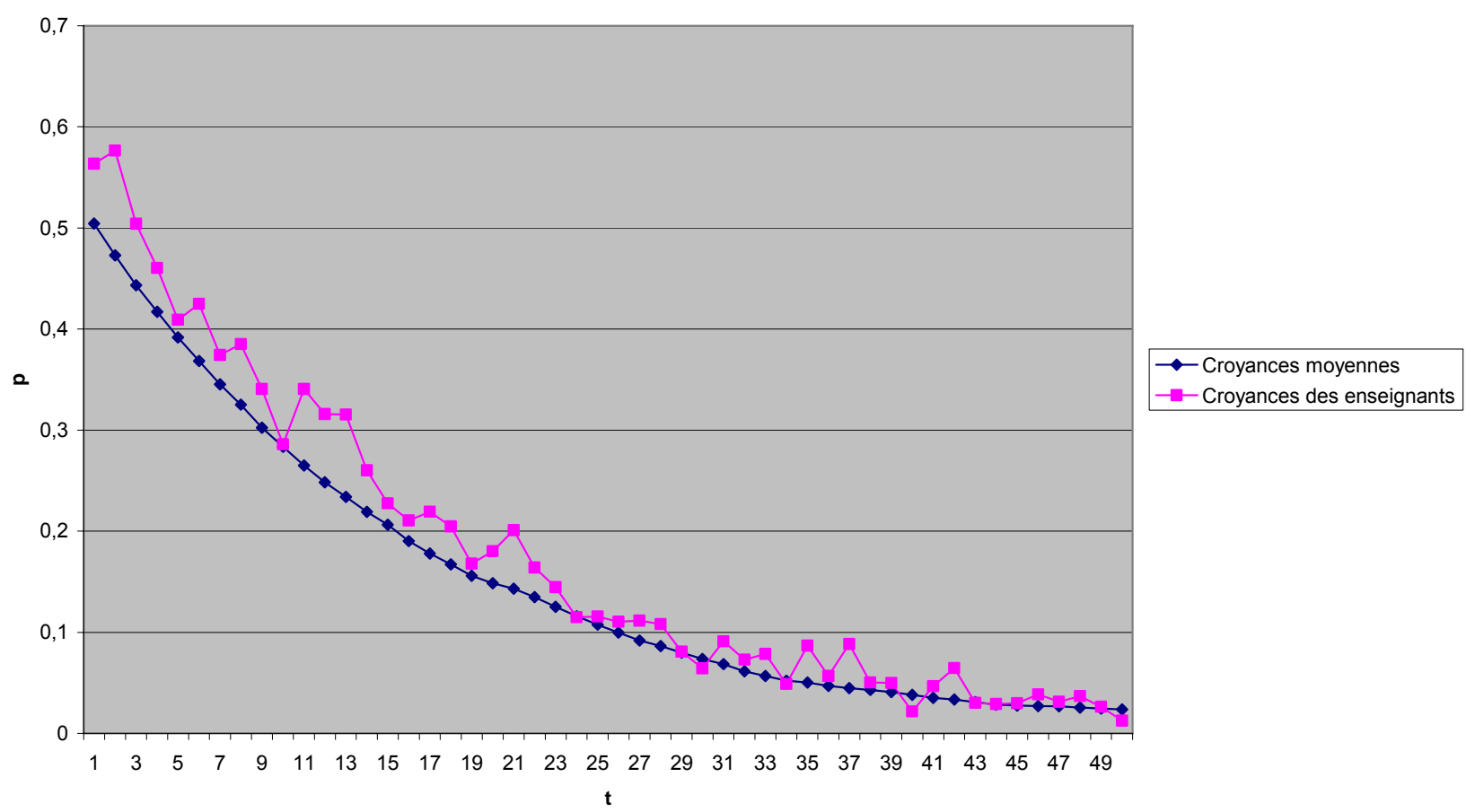

La figure 2 nous donne l'évolution de la valeur de $p$ au cours du temps lorsque l'héritabilité est totale, c'est-à-dire lorsque les enseignants n'ont aucun effet sur l'évolution des croyances, celles-ci étant entièrement déterminées par les parents. On part d'une situation initiale où les croyances sont aléatoires, uniformément distribuées sur $[0,1]$, et on converge vers $p=0$. Cette simulation a été faite avec une entropie élevée $(\lambda=2)$, le biais des enseignants est donc faible. 
La figure 3 compare l'évolution des croyances dans le cas précédent avec le cas où l'école entre pour moitié dans la formation des croyances. Il y a encore convergence vers $p=0$, mais il y a tout d'abord divergence ; la convergence est plus lente et les croyances plus hostiles à l'économie de marché.

Figure 3

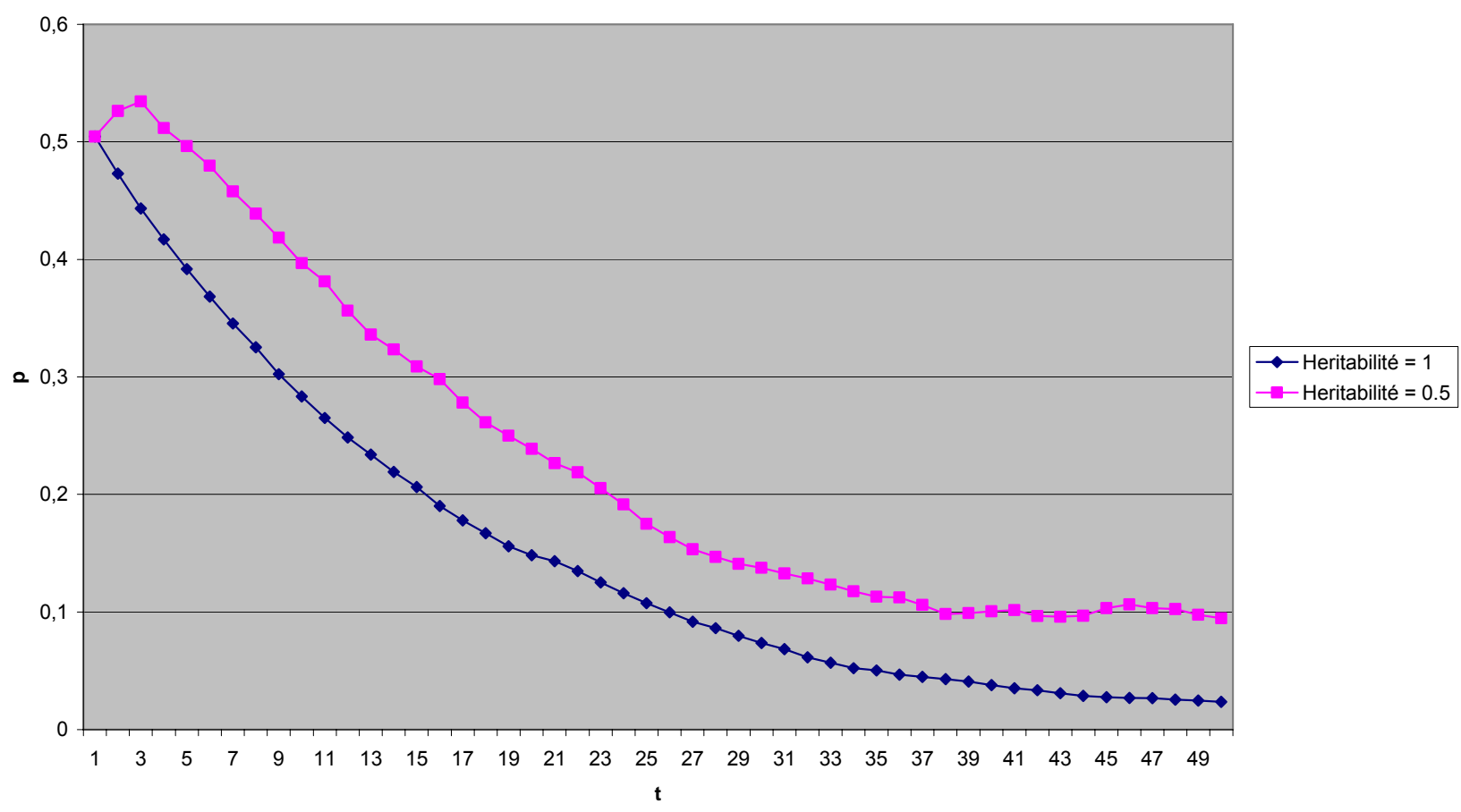

La figure 4 représente l'évolution d'une économie avec la même héritabilité de 0.5 mais une entropie beaucoup plus faible $(\lambda=50)$. Dans ce cas, le biais initial des professeurs est très élevé lors des premières générations; leurs croyances ont une forte influence sur celles de la population en général. Sur une période de 50 générations, aucune tendance à la convergence n'est observée; les individus demeurent « hostiles » à l'économie de marché. Il n'en reste pas moins que sur très longue période, l'économie converge tout de même vers $p=0$. Bien que l'entropie soit faible, une partie des agents avec un $p$ élevé ne trouve pas d'emploi dans la fonction publique, ce qui lui permet d'apprendre de façon bayésienne par 
l'expérience. En moyenne, ces agents vont contribuer à faire baisser la valeur de $p$, non seulement dans la population mais aussi chez les professeurs, ce qui contribue à la convergence de l'économie vers $p=0$.

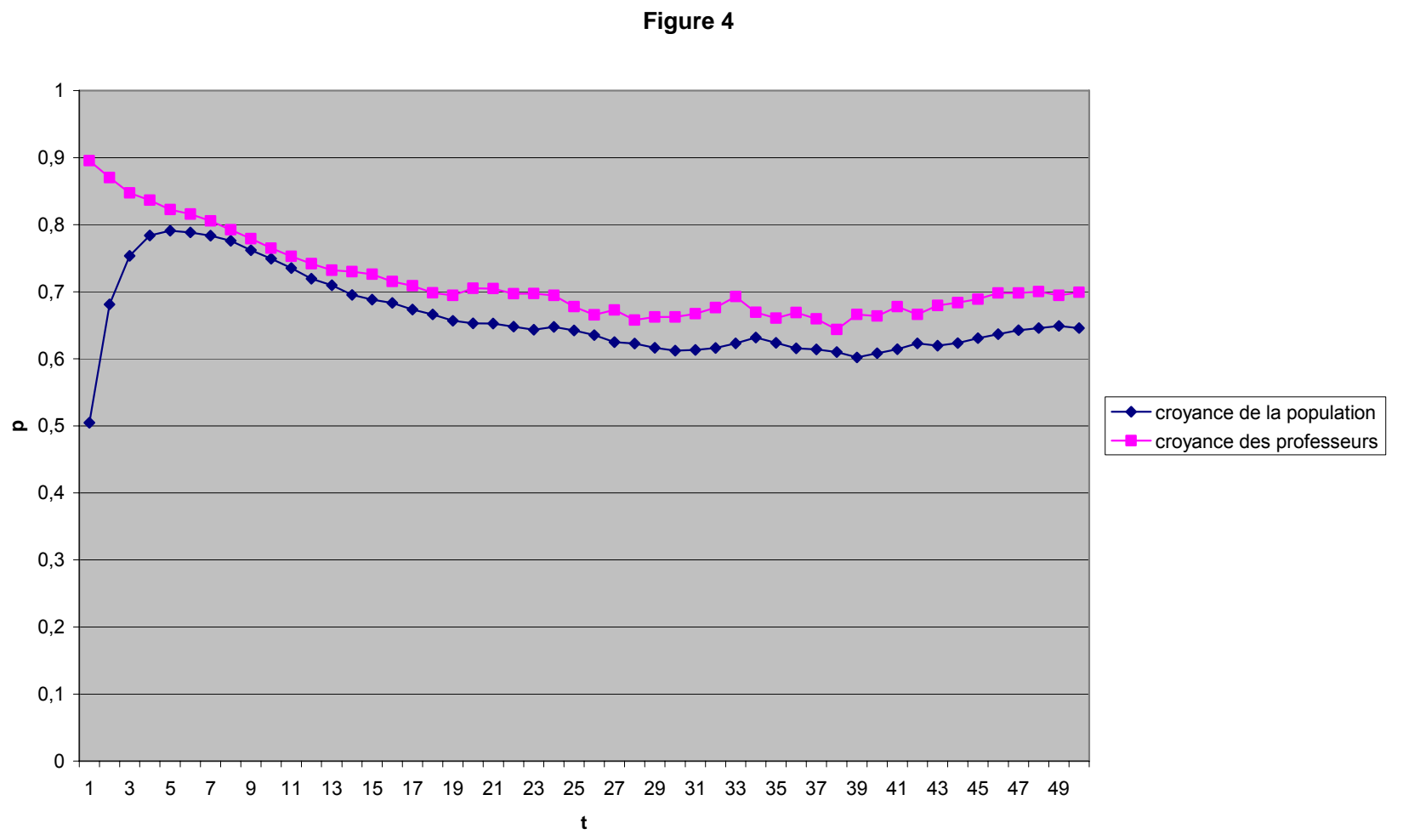

De manière générale, le biais du système éducatif ne semble pas pouvoir empêcher la convergence des croyances vers des croyances correctes. Mais, comme on vient de le voir, il peut la ralentir considérablement.

De plus, il existe des cas où la convergence n'a pas lieu.

Supposons que l'entropie soit nulle ( $\lambda$ infini) ; Comme on peut le voir sur la figure 5, l'économie converge alors vers $p=1$, c'est-à-dire les croyances les plus erronées possible et les plus «hostiles » à l'économie de marché. L'absence de mobilité intergénérationnelle chez les enseignants joue un rôle-clé dans ce résultat. La distribution des croyances chez les enfants d'enseignants reflète celles que leur a 
inculquées l'école ainsi que celles de leurs parents, qui sont également enseignants. La croyance moyenne des enfants d'enseignants est donc la même que celle des enseignants, et lorsque l'entropie est non nulle c'est le fait qu'une fraction d'entre eux ne pourra travailler dans la fonction publique qui, en les forçant à apprendre sur le tas, crée une force de rappel vers $p=0$ dans la dynamique d'apprentissage. Cependant, si l'entropie est nulle, un enfant d'enseignant ne pourra accéder à la fonction publique que si un autre agent a « surenchéri » pour ce poste. Mais pour que cela soit le cas, il faut que cet autre agent ait des croyances encore moins favorables à l'économie de marché (ce qui n'est possible que si le «prior» de ses parents était relativement proche de la valeur critique au-delà de laquelle ils seraient devenus enseignants, et s'ils ont eu une expérience négative sur le marché du travail). Cette surenchère ne contribue donc pas à réduire la valeur moyenne de $p$ pour les enseignants, mais au contraire à l'augmenter.

Figure 5

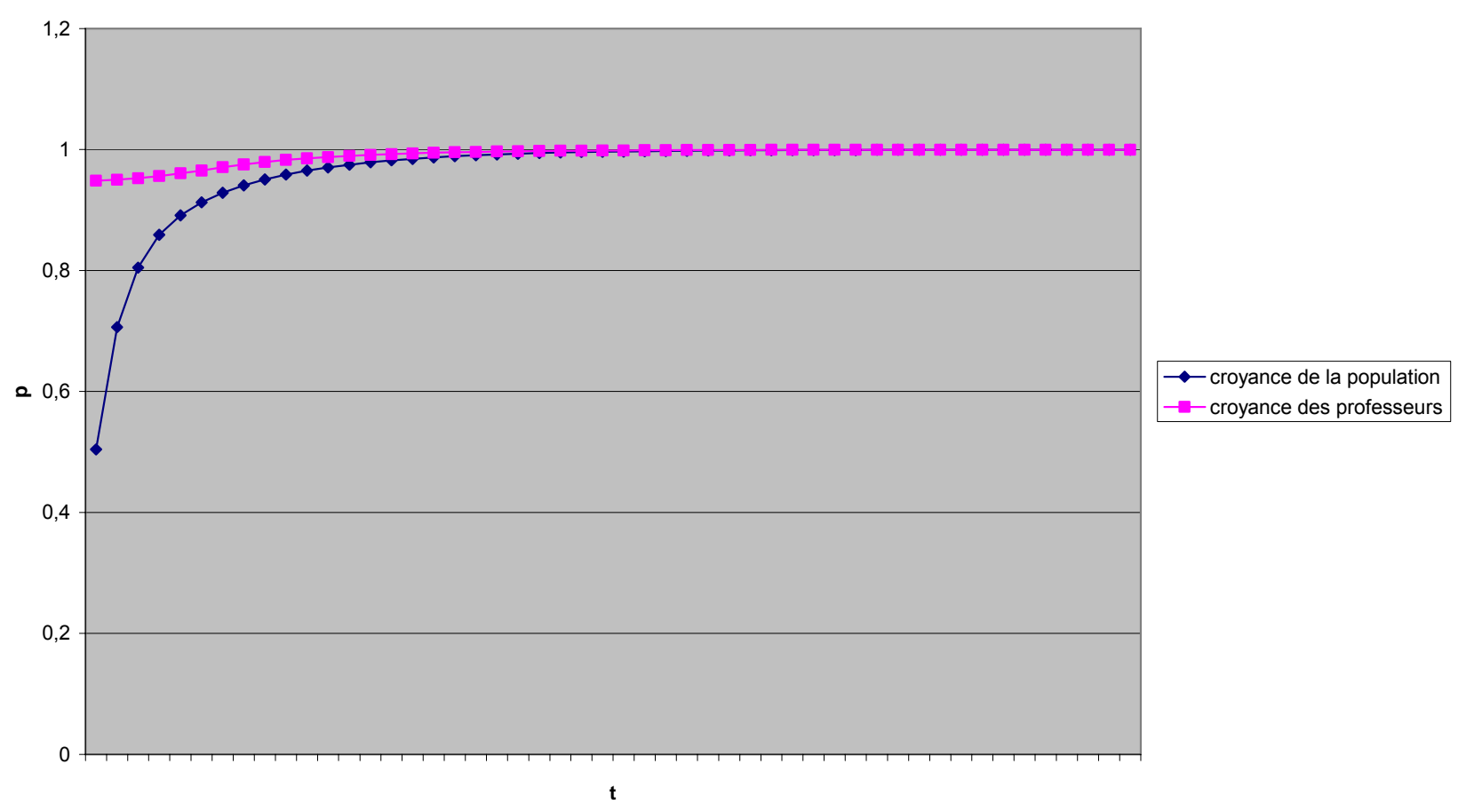

Lorsque l'héritabilité est nulle, la famille ne joue plus aucun rôle dans la transmission des croyances. Les croyances d'une nouvelle génération sont celles de 
leurs professeurs. Les professeurs de la génération suivante sont recrutés parmi les plus « pessimistes » de la nouvelle génération. On voit facilement, dans ce cas, que n'importe quelle valeur de $p$ commune à tous les individus peut être un équilibre. En d'autres termes, les croyances de long terme sont arbitraires. En effet, cette croyance sera transmise à la génération suivante intacte par les professeurs, puisque les seuls qui ont pu la réviser en fonction de leur expérience du marché ne peuvent la transmettre à leurs enfants. Les simulations numériques montrent que, dans ce cas, la distribution de $p$ dans la population converge vers une valeur de long terme commune à tous les agents, qui dépend entièrement des conditions initiales. On a donc affaire à un «effet papillon» et deux sociétés dont les croyances diffèrent peu au début peuvent converger vers des équilibres de long terme aux croyances très distinctes. Cependant, il existe une distribution de ces croyances de long terme : à cause du biais des enseignants vers un $p$ élevé, celui-ci a plus de chances d'être proche de 1 que de 0 . La figure 5 nous la décrit dans le cas d'une entropie élevée $(\lambda=0.5)$. On constate que dans la plupart des cas, l'économie converge vers une valeur de $p$ supérieure à $70 \%$. Pour une entropie faible $(\lambda=5)$, on trouve que $92 \%$ des valeurs sont comprises entre 0,9 et 1 et $8 \%$ entre 0,8 et 0,9 . Pour conclure: en l'absence d'influence familiale les croyances de long terme dépendent des événements historiques et sont d'autant plus « hostiles » à l'économie de marché que la sélection des enseignants est précise. 


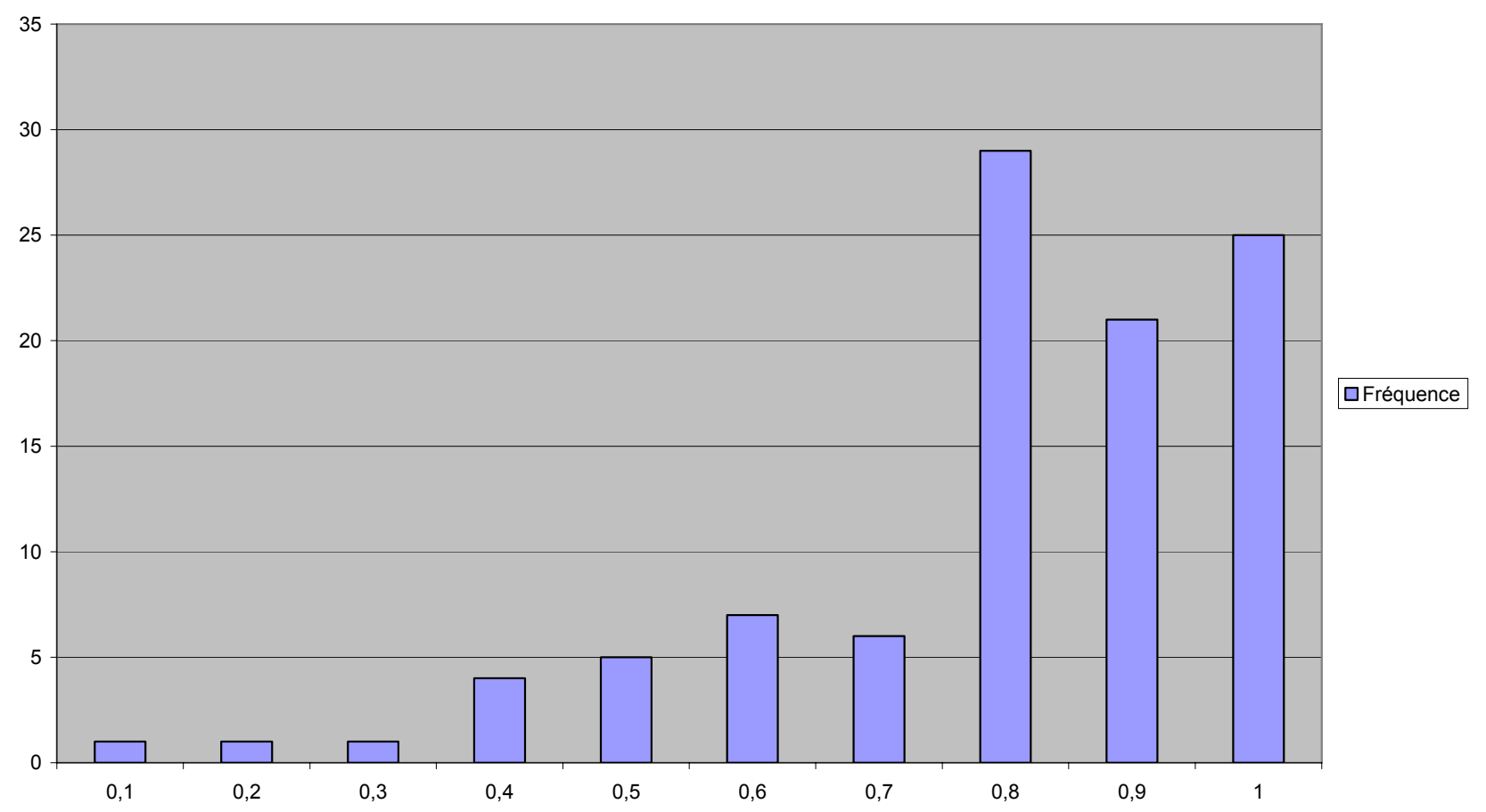

Lorsque l'entropie et l'héritabilité sont toutes deux très faibles, nos simulations suggèrent à nouveau que les croyances ne convergent pas. Ainsi, pour une héritabilité de $20 \%$ et $\lambda=50$, la valeur moyenne de $p$ à long terme fluctue entre 0.55 et 0.65 .

D'un point de vue empirique, on peut penser que l'héritabilité a baissé au cours des décennies récentes. Les progrès de la scolarisation et la hausse de l'emploi féminin tendent à réduire le temps passé par les enfants dans leurs familles et à augmenter celui passé dans des structures de type scolaire.

En ce qui concerne l'entropie, elle est plus difficile à évaluer. On pourrait la mesurer au moyen de la distribution du vote des enseignants et étudier l'évolution de cette distribution au cours du temps. On peut également étudier la mobilité intergénérationnelle des enseignants. A cet égard, Devineau et Léger (2001) montrent que la probabilité de devenir enseignant est de 25 à $35 \%$ pour les enfants

\footnotetext{
${ }^{6}$ Sophie Devineau et Alain Léger (2001), "Effet du statut professionnel du père, de la mère et du couple sur le devenir des enfants selon leur sexe: le cas des enseignants", in Education et Stratification, Caen: MRSH, juin 2001, pp. 29-68, Coll. "Cahiers de la MRSH de Caen", n² 27.
} 
d'enseignants, contre environ $15 \%$ pour les enfants de cadres supérieurs, $6 \%$ pour les enfants de patrons et de l'ordre de 3 à $5 \%$ pour les enfants d'agriculteurs ou d'ouvriers. Ces données datent de 1997 et les auteurs n'analysent malheureusement pas leur évolution au cours du temps. Mais elles recoupent celles du sondage Sofres ci-dessus en suggérant que l'entropie est faible.

Ces considérations suggèrent que les conditions sont réunies en France pour la persistance de représentations sociales défavorables à l'économie de marché à travers le système éducatif.

\section{Conclusion}

Cet article s'est intéressé à certains aspects de la persistence des idéologies et du rôle qu'elles pourraient jouer dans l'opposition de l'opinion française à la flexibilité et plus généralement à l'économie de marché. De nombreuses directions de recherche restent bien entendu à explorer, notamment en ce qui concerne le rôle des médias. 